\title{
Impact Behavior of a Rotating Rigid Body with Impact and Viscous Friction
}

\author{
Dorian Cojocaru (iD) ${ }^{1}$ and Dan B. Marghitu $\mathbb{D}^{2}$ \\ ${ }^{1}$ Department of Mechatronics and Robotics, University of Craiova, Craiova, Romania \\ ${ }^{2}$ Department of Mechanical Engineering, Auburn University, Auburn, AL 36849, USA \\ Correspondence should be addressed to Dorian Cojocaru; cojocaru@robotics.ucv.ro
}

Received 27 July 2020; Revised 11 September 2020; Accepted 17 October 2020; Published 3 November 2020

Academic Editor: Stylianos Georgantzinos

Copyright (c) 2020 Dorian Cojocaru and Dan B. Marghitu. This is an open access article distributed under the Creative Commons Attribution License, which permits unrestricted use, distribution, and reproduction in any medium, provided the original work is properly cited.

\begin{abstract}
The impact between a rotating link and a solid flat surface is considered. For the impact, we consider three distinct periods: elastic period, elastoplastic period, and restitution period. A Hertzian contact force is considered for the elastic period. Nonlinear contact forces developed from finite element analysis are used for the remaining two phases. The tangential effect is taken into account considering a friction force that combines the Coulomb dry friction model and a viscous friction function of velocity. Simulations results are obtained for different friction parameters. An experimental setup was designed to measure the contact time during impact. The experimental and simulation results are compared for different lengths of the link.
\end{abstract}

\section{Introduction}

The study of the mechanical impact has a very long history starting from Maclaurin, Newton, and Poisson. They have produced impressive number of studies for different applications, and a diversity of practical benches were used to verify the theoretical approaches.

An important improvement for this technical domain was produced through the development of new modern fields of engineering as computer aided design, robotics, and biomechanics. Modern sensors, high speed image acquisition, and processing devices and increased computer resources better support the ability for practical verification of the theoretical models. The diversity of applications must take into consideration the shape of impacting bodies, the materials, and the dynamics conditions including angles, speeds, accelerations and frictions.

Different surfaces for the impact were considered: rod with a flat surface[1], sphere with a flat [2,3], rotor system into a film damper [4], elastic wedges [5], sphere on a beam [6], anisotropic bisinusoidal surface and a rigid base [7], cylindrical body with a rigid plane [8], superball [9], tennis ball with a racket [10], and 3D-printed polymers under collision with a rigid rod [11]. Different types of behaviors depending of the nature of the constituent materials of the impacting bodies were studied: elastoplastic impact $[2,3,6,8,12-14]$, nonlinear elastic $[1,15]$, elastic [16], elastic-perfectly plastic [17], and nearly complete elastic $[14,18]$. The material properties were studied from the point of view of the influence of the impact behavior: incompressible isotropic [5], nanoparticles [15], 3D-printed polymers [11], transmembrane domains [19], stable $\mathrm{CuO}$ nanoparticle enhanced lubricants [16], Al-6061 instrumented spherical microindentation and microstructurally graded samples [20], and various finishes [21]. The direction of the impact is another factor to be considered in modeling the impact: normal [6], oblique [10,13,22], normal, and tangential [7].

Friction could have an important effect in many practical applications and by consequence the theoretical model must be adapted. Studies have considered the effect of friction in combination with other influences such as strain hardening [12], wear [15], lubrication [16], and deformation [22]. Better solutions for applications involving impacts with friction were proposed as the treatment of impact for the 
case of planar multibody mechanical systems [23] and the use of an efficient contact detection algorithm [24].

A friction model was proposed in order to make possible a correct detection of slipping, stop sliding, and reverse slipping [25]. Both cases of open and closed loop are analyzed. In order to avoid the use of Newton's hypothesis, Poisson's kinetic coefficient of restitution is proposed. Cartesian coordinates and Cartesian momenta are utilized. The final velocities are calculated momentum equations for impact. The theory is verified on some practical applications: the impact of a two-link pendulum and the impact of automotive back wheel.

The dynamics of a class of multibody mechanical systems, involving contact and friction, was also analyzed [26]. The authors assume as main contribution the development of a new method for detecting contact among the components of a system of solid bodies. The geometry of impacting system is very important from the method used to solve the problems. Analytical and numerical calculations are used for simple and complex geometries. The needed information is related to the force arising between the contacting bodies. The new method was tested by practical applications, and a corresponding methodology was proposed. A comparison between the sliding and rolling friction was performed by Alaci et al. [27]. Following the Amonton-Coulomb law, the proportionality between the friction force and the normal force was assumed for the case of the sliding friction. The dependency between the rolling friction and the normal force has been demonstrated to be linear only for specific domains. A technique has been determined in order to obtain the critical value of the coefficient of rolling friction.

A specialized software has been used for the friction behavior in Ghaednia and Jackson [15]. Specific tribology application has been targeted, but the results were applicable for friction modeling under the conditions of industrial sheet metal forming processes. Considering the influence of the temperature, Nevmerzhitskiy et al. [28] developed a friction model of industrial robot joint.

For the stance phase of the human gait, estimation for the friction force, considering both dry and viscous components, was studied by Oje and Mayo [29]. The authors stated that it is very important to have a correct estimation for the contact parameters, both normal and tangential. The researchers still look for the best estimation of tangential forces. They did not use the components of the tangential force but the modulus of this force.

Zobova [30] considered the dry friction for an element in motion on a plane with friction. In this application, the plane suffers a deformation during the motion. In order to obtain the resulting Coulomb friction force, an integration process was developed for the infinitesimal components. For this case, the estimation of normal force reactions must take into consideration the position, the orientation, the mass center velocity, and the rotation of the element.

The problem of the elastic impact is considered by Thornton et al. [31]. The application deals with spheres obliquely impacting a plane, a wall. The authors propose four different contact force models and compare the obtained results. The goal is to calibrate the normal spring stiffness by using a tangential to normal spring stiffness. The conclusion was that a linear spring can support very good results, and a nonlinear spring asks for incremental computation. A similar approach has been developed by Palli et al. [32].

A model for a single-link flexible robotic arm is studied by Chapnik et al. [33]. The model took into consideration the effects of beam damping and hub inertia. The Coulomb and viscous hub friction are computed. The model should be used also in applications with elastic impacts.

An application where face seals are designed to be in contact at standstill is presented in Green [34]. The viscous and dry frictions heating are the cause of the deformation of the seal faces. In order to obtain both, a stable operation and a favorable dynamic behavior, it is needed that the upper deformations form a converging gap for radial flow. The proposed solution is based on an elastoplastic contact model. A viscous heating model was used to compute the timedependent deformation. A plastic deformation behavior was studied by $\mathrm{Fu}$ [35].

Verifying through simulation of the abovementioned conclusions, we are proposing a trustful mathematic support for the design of mechatronics and robotic systems subjected to collisions during service. Taking into consideration the effect of friction should contribute to increasing the precision and safety for the corresponding applications. The mechanical impact study is important for robotic applications, including robotic systems for health applications. In this case one of the impacting bodies is a robotic link. The authors of this paper acquired an important experience studying different such cases as kinematic of rebound $[36,37]$, the impact between a rotating link and a massive surface [38], the impact between a planar link and a granular material [39], simultaneous impact of two links chain [19], and the variation of the coefficients of restitutions for different type of impact $[26,40,41]$.

Using the computer controlled wheelchair people with special needs can be supported also by a robotic arm picking up objects, turning on or off switches, opening or closing doors, making tea, and pulling drawers [42]. Taking into account the particularities of such applications, it is necessary to take into account the mechanical impact when designing robotic structures. In this way an adapted mechanical structure will be obtained, but it will also be possible to design and implement a more efficient control system. In order for the evaluation of the real situation to be as efficient as possible, the consideration of friction is an important factor.

The results presented in this paper were not intended for a particular robotic application but are useful to support the design process in several types of robotic applications. Some of these applications will be presented below.

The obtained results are useful for supporting the design of robotic arms that involve coupling with a pendulum [43].

The study of balance behavior is interesting for several types of mobile robots. The intrinsic ability of a robotic structure, including those that use couplings such as those studied in this paper, to function in a controllable manner, both in the absence of collisions and in the presence directly influences the overall performance of the system. A 
significant application for this aspect is the simplest case of a self-balancing mobile robot. In this situation, 2D balancing is desired for a flat robot with a double pendulum that makes a single point contact with a support surface [44].

By copying the abilities of some living creatures (e.g., a dog [45]), robotic structures have been designed that can make jumps to overcome obstacles. To make a jump, the robot must be designed in such a way that it can go through three successive actions: take-off, evolution in the air, and landing. The corresponding robotic structures are based on mechanisms that include structures such as the one studied in the paper for the implementation of hip, knee, and ankle joints.

Robots with navigational skills on terrain with complex geometry can be designed modularly. The performance of the modular design can be improved by increasing the number of modules, including modules such as the one presented in the paper [46]. Sustained research efforts are being made for the use of robotic structures in medical applications where knee prostheses, hand prostheses, and exoskeletons are needed [47]. These robotic structures include couplings of the type studied in this paper. A representative example is when people with special needs who use wheelchairs also benefit from exoskeletons for walking assistance. In this way, these people can sit, walk, and climb stairs.

Most of the applications presented above assume by their nature the existence of collisions and the influence of friction. The robotic structures can be controlled by people with special needs by manipulating a joystick but also by eye gaze [48], by gesture [49], by head motion [50], or by signals made with the fingers. In these applications it is absolutely necessary to seriously treat collisions between controlled robotic structures and obstacles.

The main objective of this paper is to propose a model for the mechanical impact, meant to support a more accurate design procedure for mechatronics and robotics systems. We present a mathematical model taking into consideration the viscous friction during impact. The relations between the impact and the friction are presented. Simulation and experimental results are developed.

\section{Mathematical Model}

For this research, in order to study the impact between a compound pendulum and a flat surface, we use a Coulomb model for the case of viscous friction. To the best of our knowledge, this frictional model has not been employed for the impact problems.

In Figure 1, a compound pendulum during the impact is represented, where $\phi$ is the angle of impact. It is needed to define a three-axis inertial reference frame. The compound link is moving in a plane, and we designated it as the $x y$-plane. The upper mentioned plane has two axes, the vertical one designated as $y$-axis (having the vertically direction downward as the positive sense), and the horizontal one designed as $x$-axis (having the sense from left to right as a positive sense). The unit vector of $x$-axis is 1 and the unit

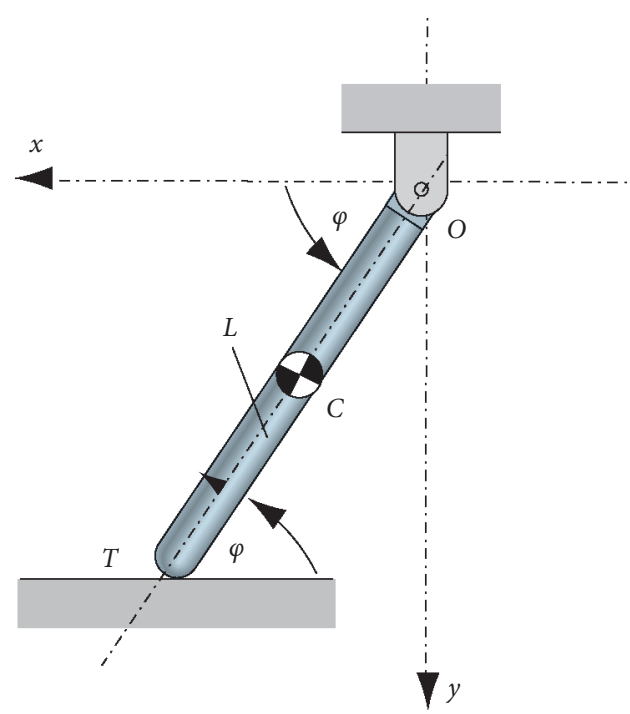

FIgURE 1: Rotating robotic link with impact.

vector of $y$-axis is $j$. The plane of motion has a normal axis, $z$, with $\mathbf{k}$ as unit vector.

Having a uniform link, the mass center $C$ has the same coordinates as the geometric center. There is a point fixed to the ground denominated the pivot point $O$. There is another point $T$ being the end of the link. This point gets in contact with a fixed plane surface. Because the length of the rotating link is $L$, the distance between the contact point $T$ and the pivot point $O$ is $L$, and the distance between the mass center $C$ and the pivot point $O$ is $L / 2$. In consequence, the vector from $O$ to $C$ is $\mathbf{r}_{O C}=\mathbf{r}=x_{C} 1+y_{C} j$, where $x_{C}$ is the horizontal coordinate of the mass center and $y_{C}$ is the vertical coordinate of the mass center $x_{C}=L / 2 \cos \phi, y_{C}=L / 2 \sin \phi$. In consequence, the position vector of $T$ is $j \mathbf{r}_{T}=x_{T} 1+y_{T} j$, where $x_{T}$ and $y_{T}$ are the coordinates of $T$ $x_{T}=L \cos \phi, y_{T}=L \sin \phi$. Hence,

$$
\mathbf{r}_{T}=L \cos \phi 1+L \sin \phi j .
$$

Taking into account that, as it was already stated, the pendulum is moving in a plane, making a pure rotation around the pivot point, it follows that the angular velocity and angular acceleration vectors follow the $z$-direction being perpendicular to this plane. We have only one moving rigid body and for the angular velocity referring to this body, the rod, we will use the following expression $\omega=\mathrm{d} \phi / \mathrm{d} t=\dot{\phi}$, where $\omega$ is the angular velocity of the rotating link. Further, making the step from the angular velocity to angular acceleration of the kinematic element, we will use the following expression: $\alpha=\mathrm{d}^{2} \phi / \mathrm{d} t^{2}=\ddot{\phi}$, where the angular acceleration of the rotating link is $\alpha$. It is needed to compute the mass moment of inertia of the rod rotating around the fixed pivot point $O$. Considering the second moment of inertia around the center of mass, one way to solve this problem is to use the transfer theorem. Thus, $I_{O}=I_{C}+m(L / 2)^{2}$. The mass of the rotating link is $m$. There is a moment supporting the rotation of the rod around the pivot point. This moment is determined by the weight of the link acting through its mass center and could be computed by the vectorial product of the 
position vector $\mathbf{r}$ crossed into $\mathbf{G}=m g j$, where $g$ is the gravitational acceleration.

At collision instant an impact normal force, $\mathbf{F}_{n}$ with the point of application at $T$ is acting upward on $y$-axis. The elastic deformation due to the normal impact force is given by

$$
\delta=L \sin \phi-L \sin \phi_{0}
$$

where $\phi_{0}$ is the initial impact angle.

The sum of the moment with respect to $O$ is sum of the vector product of the position vector $\mathbf{r}$ crossed into $\mathbf{G}$ and the vector product of the position vector $\mathbf{r}_{T}$ crossed into the impact force:

$$
I_{O} \alpha \mathbf{k}=\sum \mathbf{M}_{O}=\mathbf{r} \times \mathbf{G}+\mathbf{r}_{T} \times\left(\mathbf{F}_{n}+\mathbf{F}_{f}\right),
$$

where the friction force during impact is $\mathbf{F}_{f}$. The impact interval is divided into compression phase and restitution phase. The compression phase is initiated at the contact of the impact point with the solid surface and continues until the normal velocity of $T$ is zero. The compression phase is divided into an elastic interval and next an elastoplastic interval. The restitution interval starts at the maximum compression and ends until the impact force is zero.

\section{Impact Force}

For the normal impact force, a formulation developed by Jackson and Green [51, 52] is employed.

The Hertz theory is considered for the elastic phase during the impact and the restitution phase. The formula for the reduced modulus of elasticity is

$$
E^{-1}=\frac{1-v_{1}^{2}}{E_{1}}+\frac{1-v_{2}^{2}}{E_{2}},
$$

and the reduced radius is defined as

$$
\frac{1}{R}=\frac{1}{R_{1}}+\frac{1}{R_{2}}
$$

where $E_{1}$ and $E_{2}$ are the modulus of elasticity of the rod and the flat surface, $R_{1}$ and $R_{2}=\infty$ are the radii of curvature of the rod and the flat, and $\nu_{1}$ and $\nu_{2}$ are the Poisson ratios of the rod and the flat surface. The impact force for the elastic phase is given by

$$
F_{n}=F_{e}=\frac{4}{3} E R^{0.5} \delta^{1.5}
$$

The elastic phase is ending when

$$
\delta^{*}=\frac{\delta}{\delta_{y}}>1.9
$$

where

$$
\delta_{y}=R\left(\frac{\pi C_{j} S_{y}}{2 E}\right)^{2} \text { and } C_{j}=1.295 e^{0.736 v}
$$

The elastic displacement at which the yield starts is $\delta_{y}$ and the yield strength of the weaker material is $S_{y}$.
For the elastoplastic phase, the force is calculated with a modified Jackson and Green expression [13, 14].

$$
\begin{aligned}
F_{n}= & F_{e p}=P_{c}\left\{e^{-0.17\left(\delta / \delta_{y}\right)^{5 / 12}}\left(\frac{\delta}{\delta_{y}}\right)^{1.5}\right. \\
& \left.+\frac{4 H}{C_{j} S y}\left[1-e^{(-1 / 78)\left(\delta / \delta_{y}\right)^{5 / 9}}\left(\frac{\delta}{\delta_{y}}\right)^{1.1}\right]\right\},
\end{aligned}
$$

where

$$
\begin{aligned}
B & =0.14 e^{23 S_{y} / E}, \\
a & =\sqrt{R \delta_{y}\left(\frac{\delta}{1.9 \delta_{y}}\right)^{B},} \\
\frac{H}{S_{y}} & =2.84-0.92\left[1-\cos \left(\pi \frac{a}{R}\right)\right], \\
P_{c} & =\frac{4}{3}\left(\frac{R}{E}\right)^{2}\left(\frac{\pi C_{j} S_{y}}{2}\right)^{3} .
\end{aligned}
$$

The average normal pressure is $H$.

For the restitution, an elastic impact force is considered.

$$
F_{n}=F_{r}=\frac{4}{3} E R^{0.5}\left(\delta-\delta_{r}\right)^{1.5}
$$

where the permanent deformation is calculated from a modified curve-fitting from the finite element models $[13,22]$.

$$
\delta_{r}=\delta_{m}\left\{0.8\left[1-\left(\frac{\delta_{m}+5.5}{6.5}\right)^{-2}\right]\right\} .
$$

The maximum deformation at the end of the compression phase is $\delta_{m}$. The radius of curvature for the restitution phase is

$$
R_{r}=\frac{1}{\left(\delta_{m}-\delta_{r}\right)^{3}}\left(\frac{3 P_{m}}{4 E}\right)^{2}
$$

where $P_{m}=F_{e p}\left(\delta=\delta_{m}\right)$ is the maximum elastoplastic force.

The friction is given by a combination of the dry Coulomb force and a viscous force:

$$
\mathbf{F}_{f}=-\mu\left|\mathbf{F}_{n}\right| \frac{\left(\mathbf{v}_{T} \cdot 1\right) 1}{\left|\mathbf{v}_{T} \cdot 1\right|}-k_{v}\left(\mathbf{v}_{T} \cdot 1\right)
$$

where $\mu$ is the kinematic coefficient of friction and $k_{v}$ is the viscous coefficient of friction. The velocity of the impact point is defined as

$$
\mathbf{v}_{T}=\omega \mathbf{k} \times \mathbf{r}_{T}
$$

\section{Simulation Results}

As shown above, the purpose of the paper was to provide a solution that can support the process of designing robotic 
structures. These structures have applicability in several fields, which have also been presented above. Under these conditions, no specific customization was made for a robotic structure. One could apply the method for designing a small stepping robot that impacts a metal surface, and the impact also involves friction.

During the simulation, many experiments were performed with different conditions, giving multiple values to the quantities involved in the mathematical model. The paper included, by way of example, a particular experiment which was considered representative, but no particular solution was sought for a specific application.

To find the equations of motion, symbolical MATLAB was used. The numerical solution for the equations of motion was obtained using an ODE45 function.

The air resistance has a small value and was neglected during impact. For this application with impacts with rebounds at low velocity, the air resistance can be neglected even before and after impact.

We considered only the rigid body motion of the link. The elastic deformations were taken into account only at contact point. For future studies, we intend to include the longitudinal and transverse vibrations of the link.

Taking into account the assumed objectives and the type of robotic coupling chosen, the parameters to be calculated by simulation for the different experiments were identified.

Input variables that differentiate the experiments were chosen: geometric dimensions of the bars (length and diameter), properties of the material from which the bars are made (density, Young's modulus, Poisson ratio, and Yield strength), and nature of friction (coefficient of friction and coefficient of viscous friction).

Based on the mathematical model presented above and taking into account the behavior of the robotic couple, the kinematic and dynamic quantities for a design process were calculated for the period and point of the impact: the impact angle, angular velocity, elastic displacement, velocity (normal and tangential), kinetic energy variation, normal force, velocities of the impact point function of the coefficient of friction (tangential, normal, and angular), and rebound velocity of the impact point as a function of the viscous coefficient of friction (tangential, normal, and angular).

Considering the above, the following describes a particular experiment. The values obtained by simulation are commented in terms of variation and dependencies.

The motion of a homogeneous link, as shown in Figure 1, with the length $L=0.25 \mathrm{~m}$ and the diameter $d=2 R=0.01 \mathrm{~m}$ is simulated. The link and the flat are made of steel with the same characteristics. The density of the link is $\rho=7800 \mathrm{~kg} / \mathrm{m}^{3}$ and Young's modulus is $E=21010^{9} \mathrm{~Pa}$. The Poisson ratio is $v=0.29$ and the Yield strength is $S_{Y}=1.1210^{9} \mathrm{~Pa}$. For the first simulation, the coefficient of friction is $\mu=0.3$ and the coefficient of viscous friction is $k_{v}=80$. In Figure 2, the impact angle $\phi$ during the impact is shown. The elastic compression is very small. The elastoplastic phase is dominant and the impact angle is increasing until the maximum compression. The last phase is the restitution phase. The classical stereomechanics considered constant position during impact. The rotation of the link for

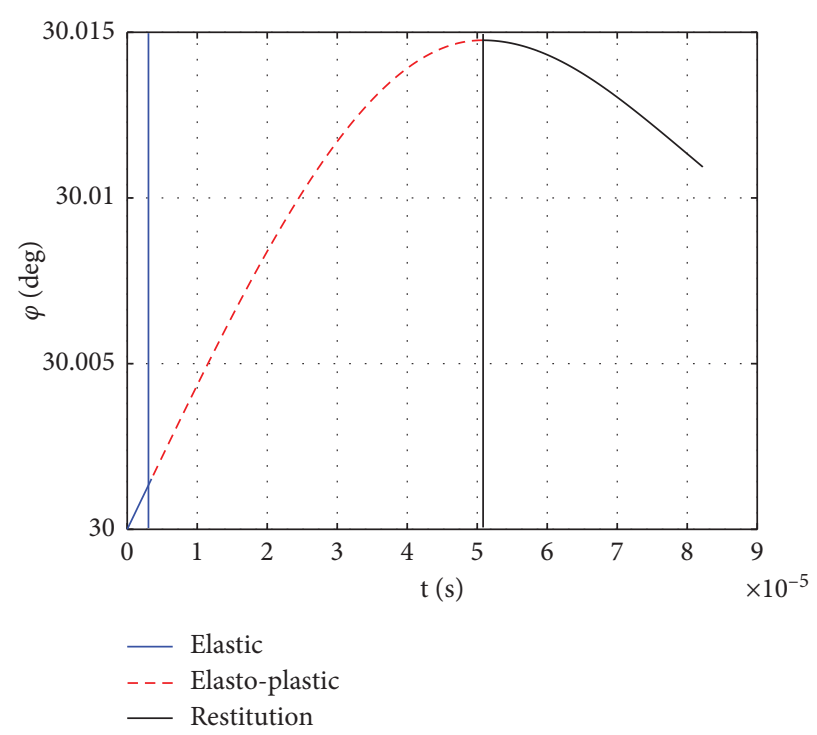

FIgURE 2: Impact angle, $\phi$, during impact.

the contact period is depicted in Figure 3. The angular velocity changes the sign at the maximum compression. The initial impact angular velocity is $\omega\left(t_{i}\right)=7.67 \mathrm{rad} / \mathrm{s}$ and the final rebound angular velocity is $\omega\left(t_{f}\right)=-3.15 \mathrm{rad} / \mathrm{s}$. At the maximum compression, the angular velocity is zero.

The elastic displacement $\delta$ is depicted in Figure 4. The maximum elastic displacement is at maximum compression and the permanent deformation is $\delta_{r}=(4.17) 10^{-5} \mathrm{~m}$. The $y$-component of the velocity of $T$ for the impact period is represented in Figure 5. The initial perpendicular velocity is $v_{T y}\left(t_{i}\right)=1.66 \mathrm{~m} / \mathrm{s}$. The final normal rebound velocity is $v_{T y}\left(t_{f}\right)=-0.68 \mathrm{~m} / \mathrm{s}$. The $x$-component of velocity of the impact point $T$ during the collision is depicted in Figure 6. The rotating element contacts the solid surface and starts sliding. The initial tangential velocity is $v_{T x}\left(t_{i}\right)=-0.96 \mathrm{~m} / \mathrm{s}$. The final tangential rebound velocity is $v_{T x}\left(t_{f}\right)=0.39 \mathrm{~m} / \mathrm{s}$. The tangential velocity changes sign at maximum compression.

The variation of the kinetic energy during impact is depicted in Figure 7. The normal force during impact is shown in Figure 8. For the next simulations, the viscous coefficient of friction is neglected. Next, the tangential velocities of the impact point as a function of the coefficient of friction $\mu$ are represented in Figure 9. The tangential rebound velocity is decreasing with the increasing of the coefficient of friction.

The normal velocities of the impact point as a function of the coefficient of friction $\mu$ are represented in Figure 10. The magnitude of the normal rebound velocity is decreasing with the increase of the coefficient of friction. If the coefficient of friction is increasing, the energy loss is increasing and the final magnitude of the rebound angular velocity is decreasing. The decreasing of the magnitude of the angular velocity during impact with the increasing of the coefficient of friction $\mu$ is shown in Figure 11.

For the next simulations, the coefficient of friction was $\mu=0.1$. In Figure 12 the rebound tangential velocity 


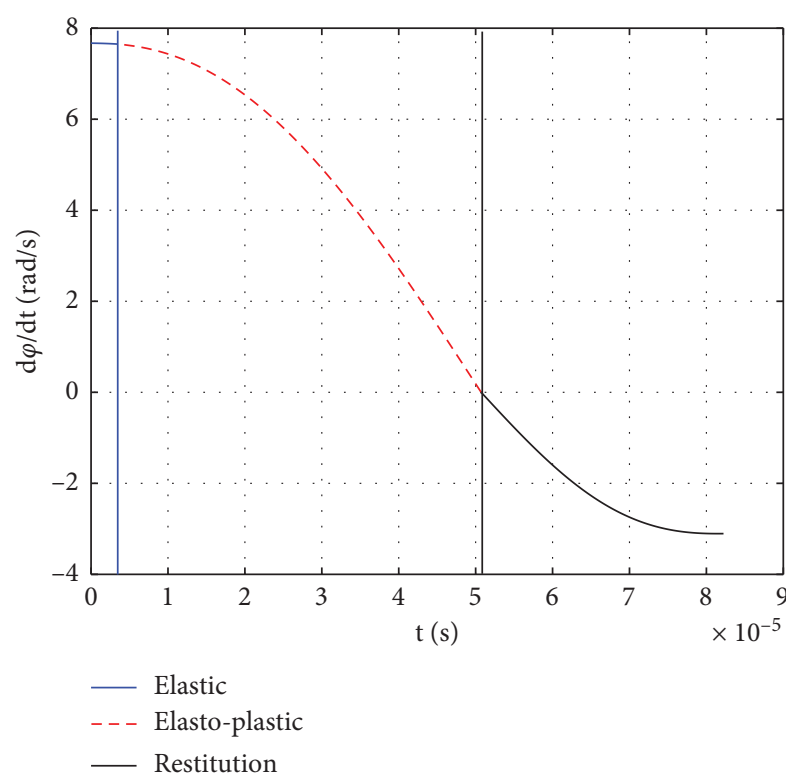

Figure 3: Angular velocity, $\mathrm{d} \phi / \mathrm{d} t$, during impact.

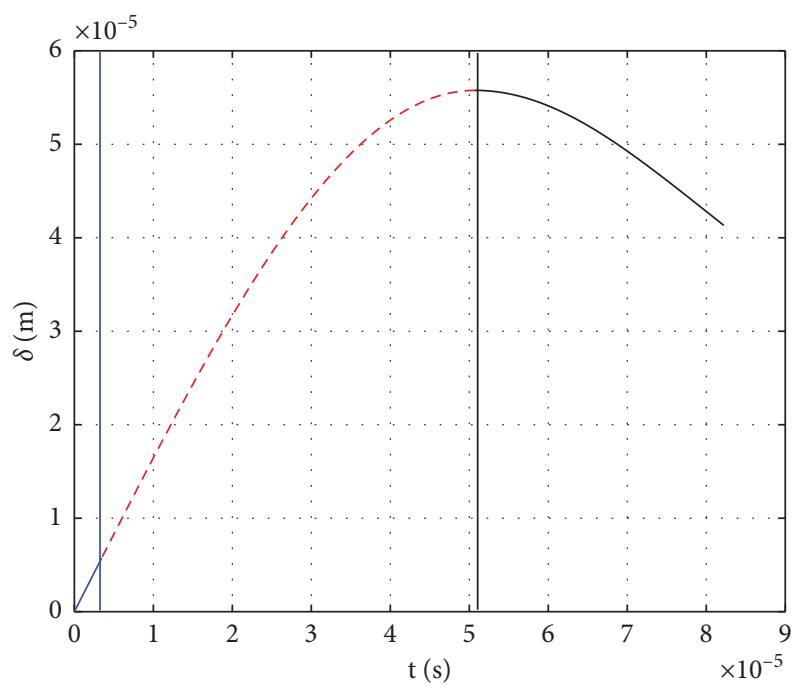

_- Elastic
- - Elasto-plastic
_ Restitution

FIgURE 4: Elastic displacement, $\delta$, during impact.

function of the coefficient of viscous friction $k_{v}$ is represented. The final tangential velocity is decreasing with respect to the viscous coefficient of friction. The magnitude of the normal rebound velocity is decreasing with respect to the viscous coefficient of friction as shown in Figure 13. The magnitude of the rebound angular velocity of the link is decreasing with respect to the viscous coefficient of friction as depicted in Figure 14.

\section{Experimental Setup}

The mathematical model was obtained by combining in a manner adapted to the experiment in question. To validate

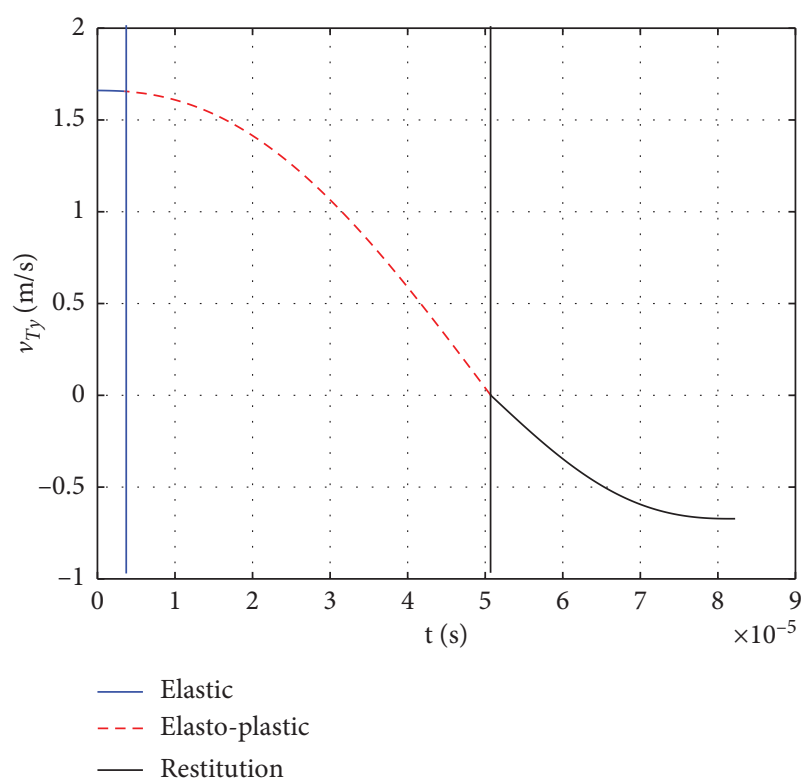

Figure 5: Normal velocity of the point $T$ during impact.

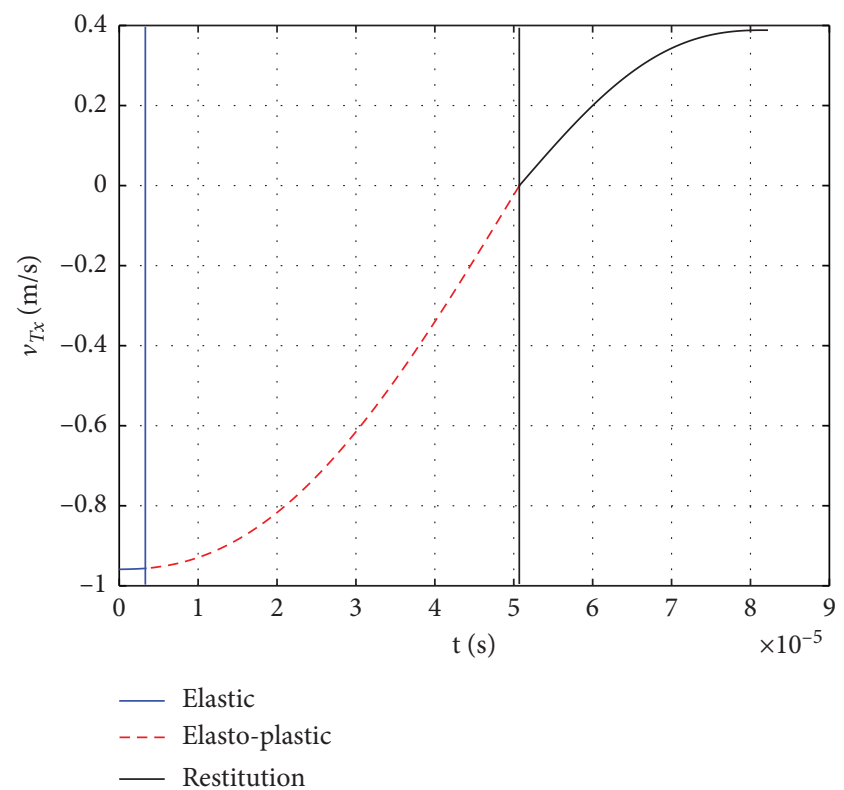

Figure 6: Tangential velocity of the point $T$ during impact.

this model, two stages were completed: numerical simulation and experimental validation.

Using the numerical simulation, the mathematical model was refined, and, using the latest version of this model, the validation was done by experimental testing. In the experimental stage, bars made of different materials and having different geometric dimensions were used.

Experimental data are acquired and stored in digital format. This data is then processed offline. As a result, processing time is not a problematic condition. Numerical simulation also does not pose problems in terms of processing cost, neither in time, nor in memory or in computing capacity. 


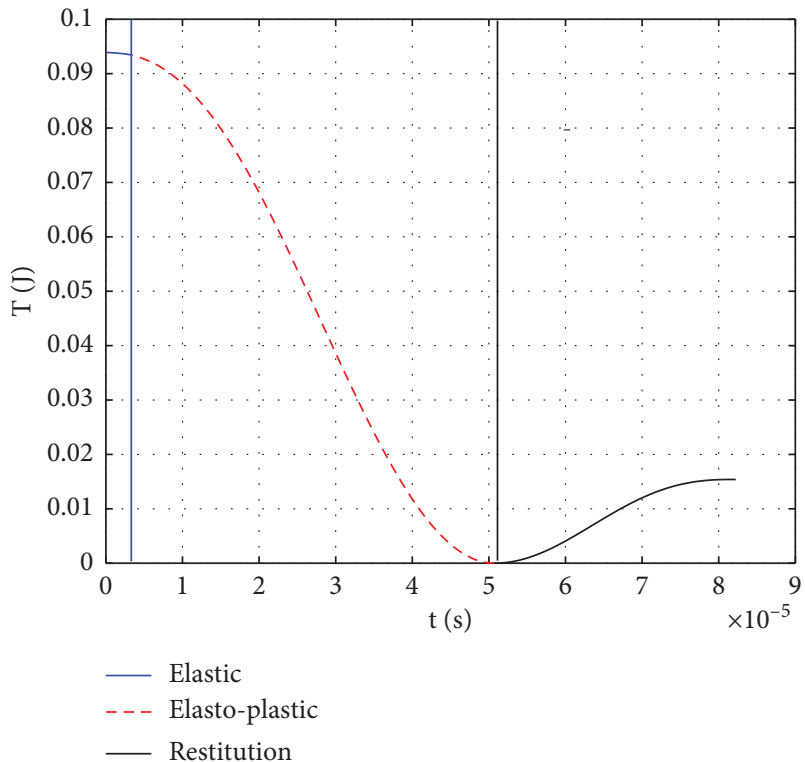

FIgURE 7: Kinetic energy variation during impact.

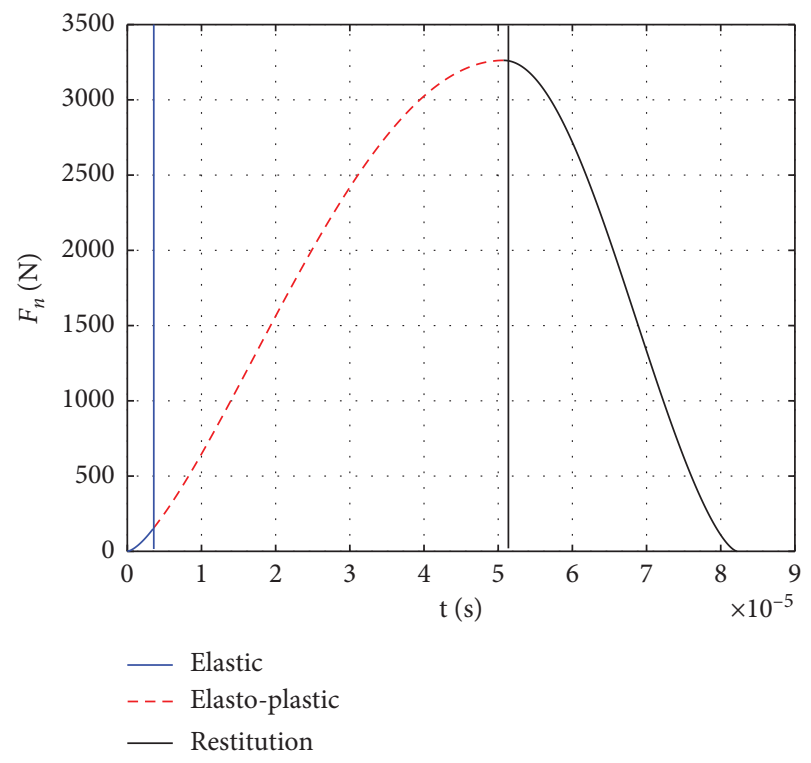

FIGURE 8: Normal force during impact.

Figure 15(a) shows the main scheme of the practical experiment. The whole structure is metallic ensuring electrical conductivity. When the coupling makes mechanical contact with the horizontal base surface, an electrical contact is also made. The resulting electrical signal is variable for a period of time due to the nature of the mechanical impact with friction. A digital oscilloscope records the signal (Figure 15(b)) which is then after that analyzed offline. To increase the accuracy of the procedure, a robotic arm with a gripper will release the coupling which, by falling, will impact the horizontal surface. A digital video system will take pictures during the fall of the couple. In this way, it is possible to determine with precision the positions in the $2 \mathrm{D}$ plane of evolution of the couple.

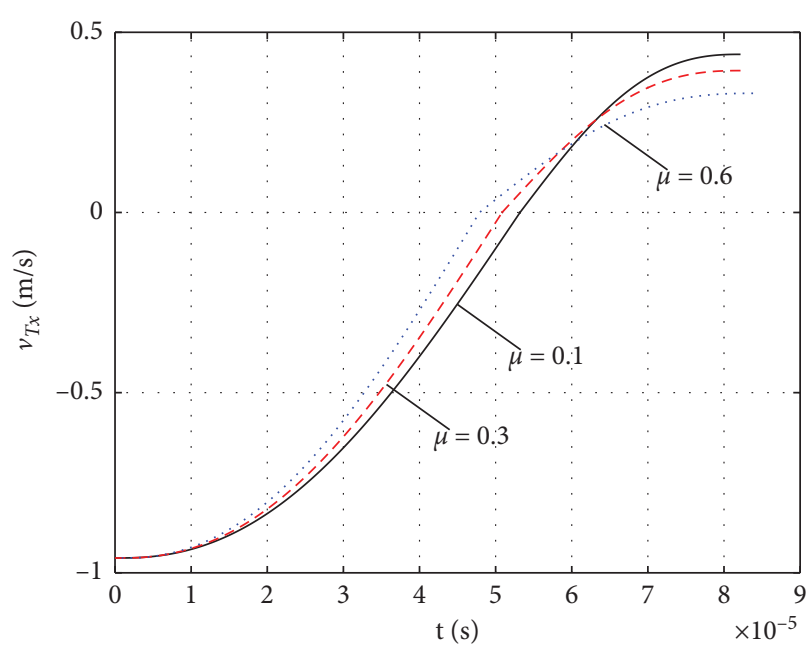

FIGURE 9: Tangential velocities of the impact point function of the coefficient of friction $\mu$.

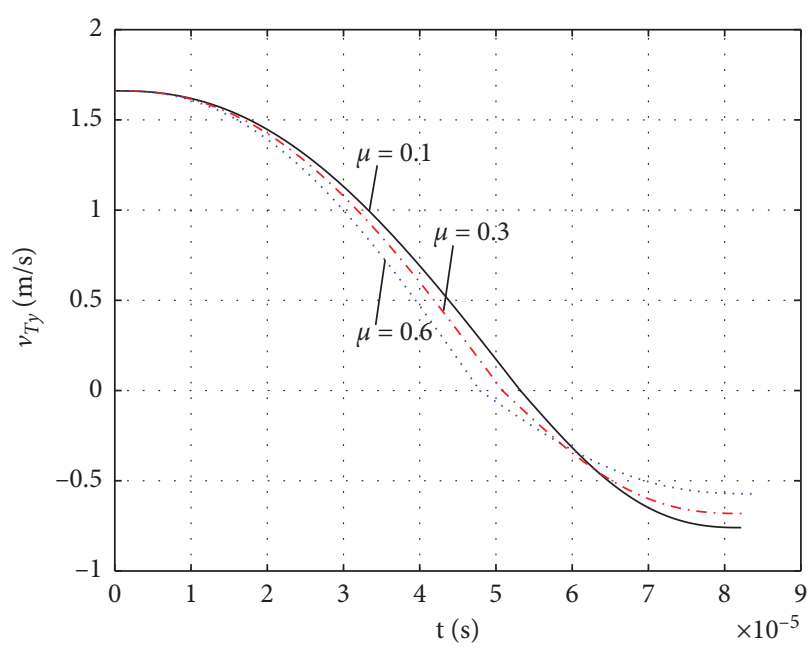

FIgURE 10: Normal velocities of the impact point function of the coefficient of friction $\mu$.

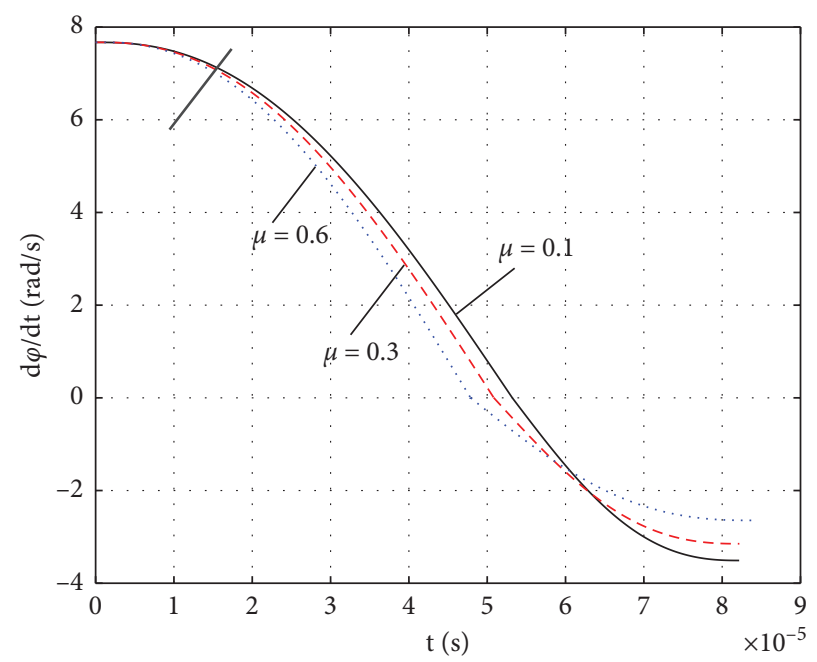

Figure 11: Angular velocity of the link as a function of the coefficient of friction $\mu$. 


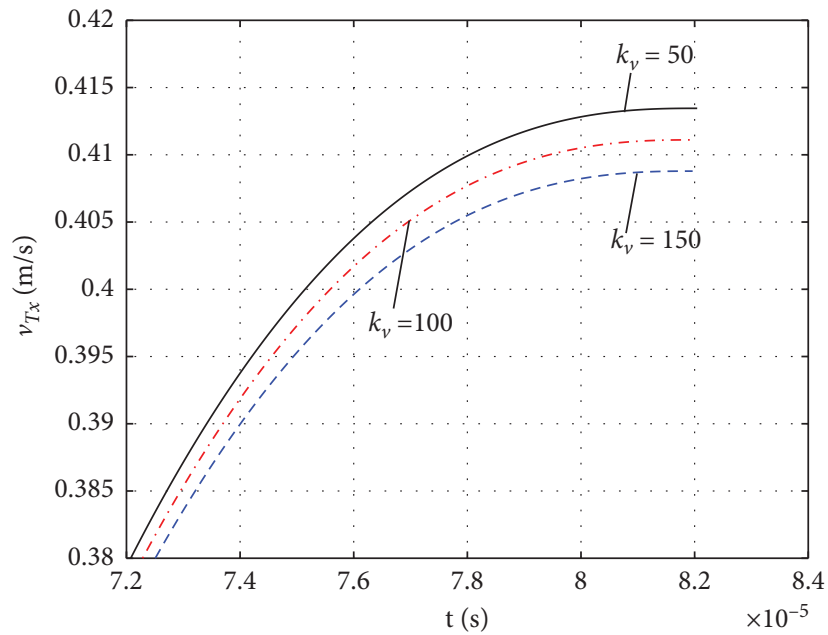

FIGURE 12: Tangential rebound velocity of the impact point as a function of the viscous coefficient of friction.

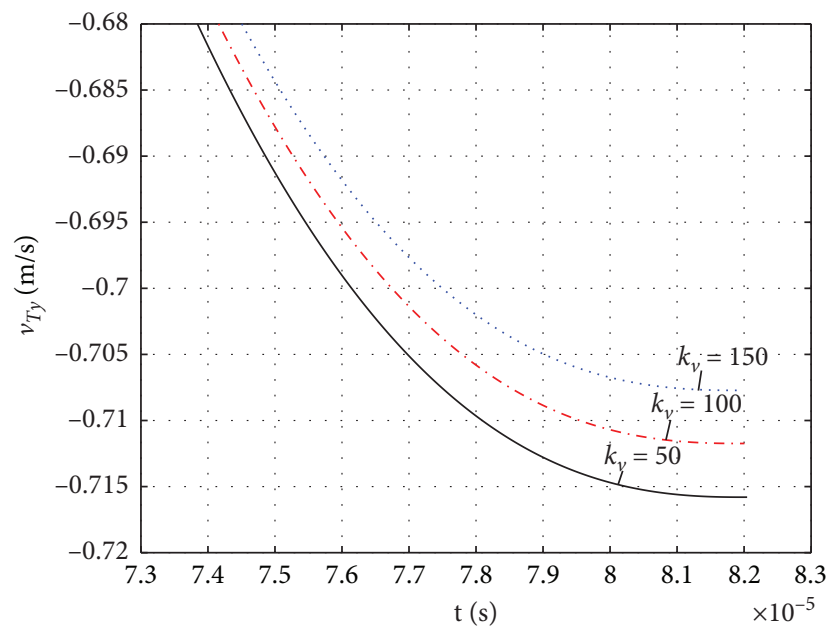

FIGURE 13: Normal rebound velocity of the impact point as a function of the viscous coefficient of friction.

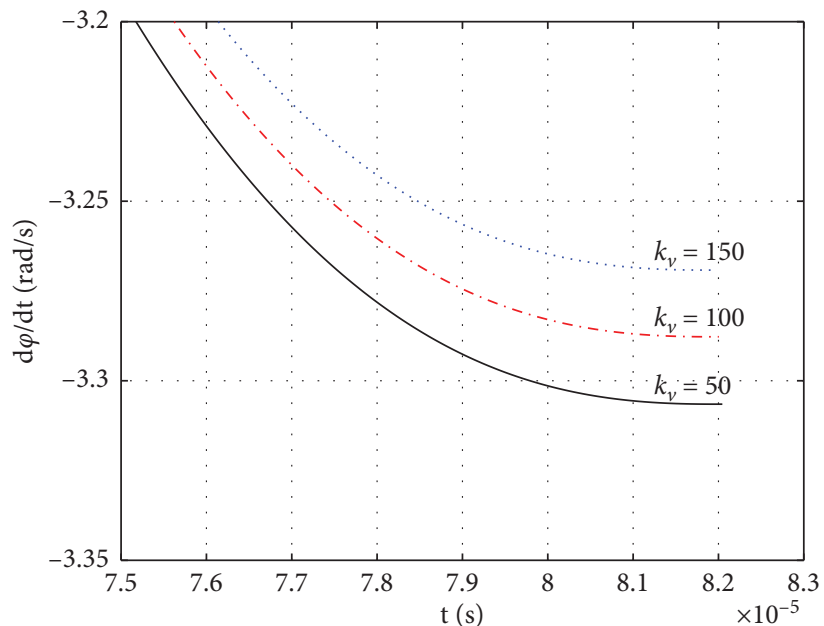

FIgURE 14: Angular rebound velocity of the link as a function of the viscous coefficient of friction. 


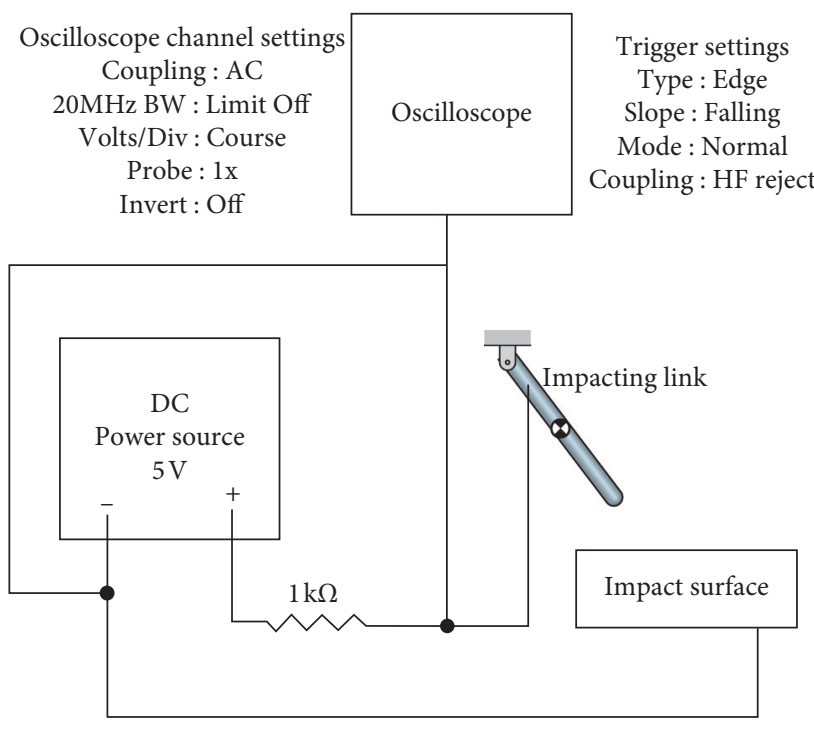

(a)

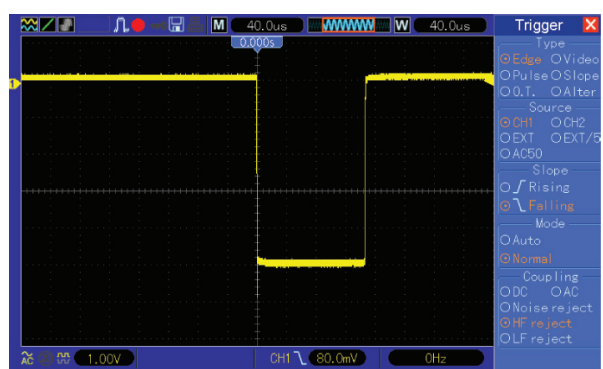

(b)

FIGURE 15: (a) Experimental setup; (b) example of an image recorded by the digital oscilloscope.

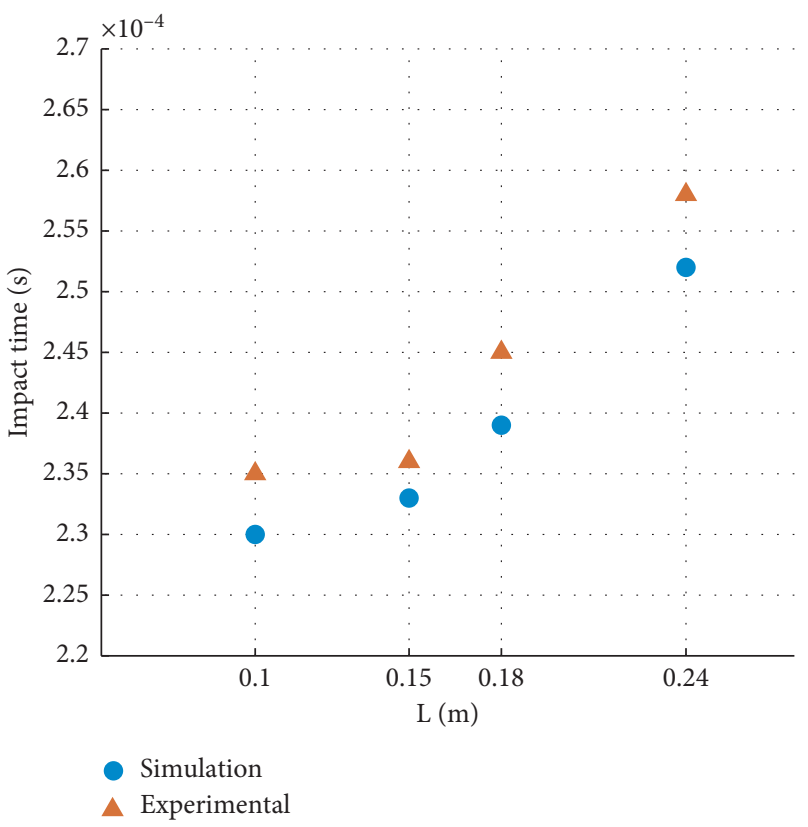

FIGURE 16: Time during impact for different lengths, $L$, of the rotating link.

The following is the information that defines one of the sets of experiments that have been performed. For the impacting link, four dimensions are used: $L_{1}=0.10 \mathrm{~m}$, $L_{2}=0.15 \mathrm{~m}, L_{3}=0.18 \mathrm{~m}$, and $L_{4}=0.24 \mathrm{~m}$. The diameter of the bars is $d=0.008 \mathrm{~m}$. The initial position of the rotating link is defined by the angle $\phi=70^{\circ}$. The bar is released from rest in a horizontal position. For the simulation, the following coefficients are used: $\mu=0.3$ and $k_{v}=100$.

In Figure 16, the mean experimental data and the simulation results are shown. The experimental and the simulation results are in good agreement. The impact time is increasing with the length of the link. For longer links, the permanent deformation is larger and the impact time will be longer.

\section{Conclusions}

The collision with viscous friction of a robotic link with a solid surface was developed. The three phases during collision are elastic compression, elastic plastic compression, and restitution. The rebound velocities were calculated function of the coefficient of friction and the viscous coefficient of friction. The rebound angular velocity of the link is increasing with respect to the viscous coefficient of 
friction. The rebound tangential velocity is decreasing with respect to the viscous coefficient of friction. To validate the theoretical model, experimental results are obtained. The duration of the impact for experimental and simulation values are similar. Verifying through simulation and experiments of the abovementioned conclusions, we are proposing a trustful mathematic support for the design of mechatronics and robotic systems subjected to collisions during service, taking into consideration that the effect of friction should contribute to increasing the precision and safety for the corresponding applications.

\section{Data Availability}

The numerical data used to support the findings of this study are included within the article.

\section{Conflicts of Interest}

The authors declare that they have no conflicts of interest.

\section{Acknowledgments}

The results presented in this paper were partially obtained in the frame of the complex research project "Intelligent and Distributed Control of 3 Complex Autonomous Systems Integrated into Emerging Technologies for Personal Medical Assistance and Precise Flexible Manufacturing Service (CIDSACTEH)," PN-III-P1-1.2-PCCDI-2017-0290, 78PCCDV/2018 at UEFISCDI, Romania.

\section{References}

[1] H. Ghaednia, D. B. Marghitu, and R. L. Jackson, "Predicting the permanent deformation after the impact of a rod with a flat surface," Journal of Tribology, vol. 137, no. 1, Article ID 11403, 2015.

[2] H. Ghaednia, S. A. Pope, R. L. Jackson, and D. B. Marghitu, "A comprehensive study of the elasto-plastic contact of a sphere and a flat," Tribology International, vol. 93, p. 7890, 2016.

[3] Z. Q. Wang, "A compact and easily accepted continuous model for the elastic-plastic contact of a sphere and a flat," Journal of Applied Mechanics, vol. 80, no. 1, Article ID 014506, 2013.

[4] H. Ghaednia and A. Ohadi A, "Vibration behavior of flexible rotor system mounted on MR squeeze film damper with thermal growth effect," Journal of Vibration and Acoustics, vol. 134, no. 1, Article ID 011015, 2012.

[5] A. Golgoon, S. Sadik, and A. Yavari, "Circumferentiallysymmetric finite eigenstrains in incompressible isotropic nonlinear elastic wedges," International Journal of Non-linear Mechanics, vol. 84, Article ID 116129, 2016.

[6] H. Wang, X. Yin, X. Qi, Q. Deng, B. Yu, and Q. Hao, "Experimental and theoretical analysis of the elastic-plastic normal repeated impacts of a sphere on a beam," International Journal of Solids and Structures, vol. 22, 2017.

[7] X. Wang, Y. Xu, and R. L. Jackson, "Elasticplastic sinusoidal waviness contact under combined normal and tangential loading," Tribology Letters, vol. 65, no. 2, p. 45, 2017.

[8] B. Zhao, F. Shen, Y. Cui, Y. Xie, and K. Zhou, "Damage analysis for an elastic-plastic body in cylindrical contact with a rigid plane," Tribology International, vol. 115, pp. 18-27, 2017.
[9] R. Cross, "Impact behavior of a superball," American Journal of Physics, vol. 83, no. 3, Article ID 238248, 2015.

[10] H. Ghaednia, O. Cermik, and D. B. Marghitu, "Experimental and theoretical study of the oblique impact of a tennis ball with a racket," Proceedings of the Institution of Mechanical Engineers, Part P: Journal of Sports Engineering and Technology, vol. 229, no. 3, Article ID 149158, 2015.

[11] K. Kardel, H. Ghaednia, A. L. Carrano, and D. B. Marghitu, "Experimental and theoretical modeling of behavior of 3Dprinted polymers under collision with a rigid rod," Additive Manufacturing, vol. 14, p. 8794, 2017.

[12] M. R. Brake, "An analytical elastic plastic contact model with strain hardening and frictional effects for normal and oblique impacts," International Journal of Solids and Structures, vol. 62, Article ID 104123, 2015.

[13] H. Gheadnia, O. Cermik, and D. B. Marghitu, "Experimental and theoretical analysis of the elasto-plastic oblique impact of a rod with a flat," International Journal of Impact Engineering, vol. 86, Article ID 307317, 2015.

[14] Y. Xu, R. L. Jackson, and D. B. Marghitu, "Statistical model of nearly complete elastic trough surface contact," International Journal of Solids and Structures, vol. 51, no. 5, Article ID 1075088, 2014.

[15] H. Ghaednia and R. L. Jackson, "The effect of nanoparticles on the real area of contact, friction, and wear," Journal of Tribology, vol. 135, no. 4, Article ID 041603, 2013.

[16] H. Ghaednia, L. R. Jackson, and J. M. Khodadadi, "Experimental analysis of stable $\mathrm{CuO}$ nanoparticle enhanced lubricants," Journal of Experimental Nanoscience, vol. 10, no. 1, p. 118, 2015.

[17] M. R. Brake, "An analytical elastic-perfectly plastic contact model," International Journal of Solids and Structures, vol. 49, no. 22, Article ID 31293141, 2012.

[18] Y. Xu and R. L. Jackson, "Statistical models of nearly complete elastic trough surface contact-comparison with numerical solutions," Tribology International, vol. 105, pp. 274-291, Article ID 274291, 2017.

[19] D. B. Marghitu and D. Cojocaru, "Simultaneous impact of a two-link chain, nonlinear dynamics," in Proceedings of the International Journal of Nonlinear Dynamics and Chaos in Engineering, Sinaia, Romania, December 2014.

[20] J. S. Weaver, A. Khosravani, A. Castillo, and S. R. Kalidindi, "High throughput exploration of process-property linkages in Al-6061 using instrumented spherical microindentation and microstructurally graded samples," Integrating Materials and Manufacturing Innovation, vol. 5, no. 1, p. 10, 2016.

[21] X. Zhang and R. L. Jackson, "An analysis of the multiscale structure of surfaces with various nishes," Tribology Transactions, vol. 60, no. 1, Article ID 121134, 2017.

[22] H. Ghaednia and D. B. Marghitu, "Permanent deformation during the oblique impact with friction," Archive of Applied Mechanics, vol. 86, no. 1-2, Article ID 121134, 2016.

[23] H. M. Lankarani and M. F. O. S. Pereira, "Treatment of impact with friction in planar multibody mechanical systems," Multibody System Dynamics, vol. 6, no. 3, pp. 203-227, 2001.

[24] A. Pournaras, F. Karaoulanis, and S. Natsiavas, "Dynamics of mechanical systems involving impact and friction using an efficient contact detection algorithm," International Journal of Non-linear Mechanics, vol. 94, pp. 309-322, 2017.

[25] H. M. Lankarani, "A Poisson based formulation for frictional analysis of multibody mechanical systems with open or closed kinematic chains," Journal of Mechanical Design, vol. 122, no. $4,2000$. 
[26] D. B. Marghitu, D. Cojocaru, and S. Dumitru, "The variation of the coefficient of restitution with the impact angle," in Proceedings of the 16th International Conference on System Theory, Control and Computing Joint Conference SINTES 16, SACCS 12, SIMSIS 16, Sinaia, Romania, October 2012.

[27] S. Alaci, F. C. Ciornei, I. C. Romanu, and M. C. Ciornei, "Upon the relationship between rolling friction and sliding friction," IOP Conference Series: Materials Science and Engineering, vol. 400, Article ID 042002, 2018.

[28] M. N. Nevmerzhitskiy, B. S. Notkin, A. V. Vara, and K. V. Zmeu, "Friction model of industrial robot joint with temperature correction by example of KUKA KR10," Journal of Robotics, vol. 2019, Article ID 6931563, 11 pages, 2019.

[29] J. Ojeda and J. Mayo, "A procedure to estimate normal and friction contact parameters in the stance phase of the human gait," Computer Methods in Biomechanics and Biomedical Engineering, vol. 22, no. 8, pp. 840-852, 2019.

[30] A. A. Zobova, "Dry friction distributed over a contact patch between a rigid body and a visco-elastic plane," Multibody System Dynamics, vol. 45, no. 2, p. 203, 2019.

[31] C. Thornton, S. J. Cummins, and P. W. Cleary, “An investigation of the comparative behaviour of alternative contact force models during elastic collisions," Powder Technology, vol. 210, no. 3, pp. 189-197, 2011.

[32] G. Palli and C. Melchiorri, "Output-based control of robots with variable stiffness actuation," Journal of Robotics, vol. 2011, Article ID 735407, 15 pages, 2011.

[33] B. V. Chapnik, G. R. Heppler, and J. D. Aplevich, "Modeling impact on a one-link flexible robotic arm," IEEE Transactions On Robotics And Automation, vol. 7, no. 4, pp. 479-488, 1991.

[34] I. Green, "A transient dynamic analysis of mechanical seals including asperity contact and face deformation," Tribology Transactions, vol. 45, no. 3, pp. 284-293, 2002.

[35] L. Fu and J. Zhao, "Design and implementation of plastic deformation behavior by cartesian impedance control based on maxwell model," Complexity, vol. 2018, Article ID 6752752, 9 pages, 2018.

[36] D. Cojocaru and D. B. Marghitu, "Experiments of rebound for robotic kinematic," in Proceedings of the International Conference on Circuits, Systems, pp. 369-373, Alberta, Canada, January 2010.

[37] D. B. Marghitu and D. Cojocaru, "Rebound of a robotic kinematic element," Annals of the University of Craiova Series: Automation, Computers, Electronics and Mechatronics, vol. 33, no. 2, pp. 30-33, 2009.

[38] D. B. Marghitu, D. Cojocaru, and R. L. Jackson, "Elasto-plastic impact of a rotating link with a massive surface," International Journal of Mechanical Sciences, vol. 53, no. 4, pp. 309-315, 2011.

[39] D. B. Marghitu, L. Seunghun, and D. Cojocaru, "Stopping time for a planar link in contact with a granular material," Annals of the University of Craiova Series: Automation, Computers, Electronics and Mechatronics, vol. 35, no. 1, pp. 21-26, 2011.

[40] M. Arsalan, H. Ghaednia, D. B. Marghitu, and D. Cojocaru, "Force analysis for the impact between a rod and granular material," Advances in Service and Industrial Robotics, vol. 49, pp. 85-94, 2018.

[41] D. Cojocaru and D. B. Marghitu, "Differential formulation for the coefficient of restitution of a rigid link," Applied Mechanics And Materials, Mechatronics And Robotics, vol. 762, pp. 175-183, 2014.

[42] H. F. N. Al-Shuka, S. Leonhardt, W.-H. Zhu, R. Song, C. Ding, and Y. Li, "Active impedance control of bioinspired motion robotic manipulators: an overview," Applied Bionics and Biomechanics, vol. 2018, Article ID 8203054, 19 pages, 2018.

[43] A. Notue Kadjie, P. R. Nwagoum Tuwa, and P. Woafo, "An electromechanical pendulum robot arm in action: dynamics and control," Shock and Vibration, vol. 2017, Article ID 3979384, 13 pages, 2017.

[44] R. Featherstone, Analysis and Design of Planar Self-Balancing Double-Pendulum, http://royfeatherstone.org/papers/ romansy2012_9.pdf.

[45] Z. Zhang, J. Zhao, H. L. Chen, and D. S. Chen, "A survey of bioinspired jumping robot: takeoff, air posture adjustment, and landing buffer," Applied Bionics and Biomechanics, vol. 2017, Article ID 4780160, 22 pages, 2017.

[46] M. Ning, L. Shao, F. J. Chen, M. Li, C. Zhang, and Q. Zhang, "Modeling and analysis of a modular multilegged robot with improved fault tolerance and environmental adaptability," Mathematical Problems in Engineering, vol. 2019, Article ID 8261617, 17 pages, 2019.

[47] R. A. Beasley, "Medical robots: current systems and research directions," Journal of Robotics Hindawi, vol. 2012, Article ID 401613, 14 pages, 2012.

[48] D. Cojocaru, L. F. Manta, I. C. Vladu, A. Dragomir, and A. Mariniuc, "Using an eye gaze new combined approach to control a wheelchair movement," in Proceedings of the 2019 23rd International Conference on System Theory, Control and Computing (ICSTCC), Sinaia, Romania, October 2019.

[49] B. Baldursson, B. Rasti, K. Gudmundsson, D. Cojocaru, K. Andersen, and S. E. Thorsteinsson, "Gesture interpretation control system using convolutional neural networks," 2019 International Conference on Biomedical Innovations and Applications (BIA), vol. 11, 2019.

[50] L. F. Manta, D. Cojocaru, I. C. Vladu, A. Dragomir, and A. Mariniuc, "Wheelchair control by head motion using a non-contact method in relation to the patient," in Proceedings of the 20th International Carpathian Control Conference, Turówka, Poland, May 2019.

[51] R. L. Jackson and I. Green, "A finite element study of elastoplastic hemispherical contact against a rigid flat," Journal of Tribology, vol. 127, no. 2, pp. 343-354, 2005.

[52] R. L. Jackson, I. Green, and D. B. Marghitu, "Predicting the coefficient of restitution of impacting elastic-perfectly plastic spheres," Nonlinear Dynamics, vol. 60, no. 3, pp. 217-229, 2010. 